\title{
The Midpoint-Constrained Egalitarian Bargaining Solution
}

Citation for published version (APA):

Karos, D., \& Rachmilevitch, S. (2018). The Midpoint-Constrained Egalitarian Bargaining Solution. Maastricht University, Graduate School of Business and Economics. GSBE Research Memoranda No. $007 \mathrm{https}: / /$ doi.org/10.26481/umagsb.2018007

Document status and date:

Published: 29/03/2018

DOI:

10.26481/umagsb.2018007

Document Version:

Publisher's PDF, also known as Version of record

\section{Please check the document version of this publication:}

- A submitted manuscript is the version of the article upon submission and before peer-review. There can be important differences between the submitted version and the official published version of record.

People interested in the research are advised to contact the author for the final version of the publication, or visit the DOI to the publisher's website.

- The final author version and the galley proof are versions of the publication after peer review.

- The final published version features the final layout of the paper including the volume, issue and page numbers.

Link to publication

\footnotetext{
General rights rights.

- You may freely distribute the URL identifying the publication in the public portal. please follow below link for the End User Agreement:

www.umlib.nl/taverne-license

Take down policy

If you believe that this document breaches copyright please contact us at:

repository@maastrichtuniversity.nl

providing details and we will investigate your claim.
}

Copyright and moral rights for the publications made accessible in the public portal are retained by the authors and/or other copyright owners and it is a condition of accessing publications that users recognise and abide by the legal requirements associated with these

- Users may download and print one copy of any publication from the public portal for the purpose of private study or research.

- You may not further distribute the material or use it for any profit-making activity or commercial gain

If the publication is distributed under the terms of Article $25 \mathrm{fa}$ of the Dutch Copyright Act, indicated by the "Taverne" license above, 


\section{Maastricht University}

Dominik Karos, Shiran

Rachmilevitch

The Midpoint-Constrained Egalitarian Bargaining Solution

$\mathrm{RM} / 18 / 007$

\section{GSBE}

Maastricht University School of Business and Economics

Graduate School of Business and Economics

P.O Box 616

NL- 6200 MD Maastricht

The Netherlands 


\title{
The Midpoint-Constrained Egalitarian Bargaining Solution
}

\author{
Dominik Karos $^{1}$ and Shiran Rachmilevitch ${ }^{2}$ \\ ${ }^{1}$ School of Business and Economics, Maastricht University, The Netherlands. \\ d.karos@maastrichtuniversity.nl \\ ${ }^{2}$ Department of Economics, University of Haifa, Israel. \\ shiranrach@gmail.com
}

March 26, 2018

\begin{abstract}
A payoff allocation in a bargaining problem is midpoint dominant if each player obtains at least one $n$-th of her ideal payoff. The egalitarian solution of a bargaining problem may select a payoff configuration which is not midpoint dominant. We propose and characterize the solution which selects for each bargaining problem the feasible allocation that is closest to the egalitarian allocation, subject to being midpoint dominant. Our main axiom, midpoint monotonicity, is new to the literature; it imposes the standard monotonicity requirement whenever doing so does not result in selecting an allocation which is not midpoint dominant. In order to prove our main result we develop a general extension theorem for bargaining solutions that are order-preserving with respect to any order on the set of bargaining problems.
\end{abstract}

Keywords: Bargaining; Egalitarianism; Midpoint domination. JEL Codes: C71; C78; D61; D63. 


\section{Introduction}

In a bargaining problem $n$ players have to agree on a feasible utility allocation: if they can't reach an agreement, they will receive zero payoffs. A bargaining solution is a map that selects a unique feasible payoff vector for every bargaining problem - as an arbitrator would do (Luce and Raiffa, 1957).

One of the simplest options this arbitrator has is to determine a "dictator" by flipping a fair coin: the selected player will receive her maximum possible payoff, while any other player receives zero. The requirement that a bargaining solution Pareto-dominate this simple procedure has been formalized by Sobel (1981) as the midpoint domination axiom. It is satisfied by the solutions of Nash (1950) and Kalai and Smorodinsky (1975) (see for instance Moulin, 1983; Rachmilevitch, 2014, respectively); but it is violated by the monotonic solutions of Kalai (1977). Even worse, we show that the only solution that is monotonic and midpoint dominant is the procedure above - which, in general, is not Pareto efficient.

We propose a variant of the egalitarian - that is, monotonic and symmetricsolution that is designed to overcome this problem: the midpoint-constrained egalitarian solution, which selects for every problem, among the points that are (coordinate wise) greater than or equal to the midpoint, the one which is closest to the egalitarian point. We characterize this solution by four axioms: weak Pareto efficiency, symmetry, midpoint domination, and midpoint monotonicity. The first three are well-known in the bargaining literature; the fourth is novel, and the requirement it imposes is as follows: when the set of feasible utility vectors expands, the only justification to lower any player's payoff is that this is necessary for avoiding a violation of midpoint domination. In other words, all players should benefit from the addition of alternatives, unless this would imply a violation of midpoint domination.

Technically, we proceed in two steps. First, we characterize our solution on the class $\mathcal{B}^{*}$ of problems in which the weak and strong Pareto frontiers coincide. We then prove an extension theorem for bargaining solutions: A bargaining solution is 
weakly monotonic if it is order-preserving with respect to some (arbitrary) order on the set of bargaining problems. Our theorem states that a bargaining solution that is continuous and weakly monotonic is already uniquely determined by its values on $\mathcal{B}^{*}$.

The rest of the paper is organized as follows. Section 2 introduces some notation and standard axioms. Section 3 starts by characterizing the unique solution that satisfies monotonicity and midpoint domination, which is not Pareto efficient. This negative result motivates our midpoint monotonicity axiom that we propose at the end of that section. We present and analyze our new solution in Section 4. The extension theorem and its application are in Section 5. Section 6 contains a brief discussion.

\section{Preliminaries}

\section{$2.1 \quad$ Notation}

Let $N=\{1, \ldots, n\}$ be a finite set of players. Let $\overline{\mathcal{B}}$ be the collections of pairs $(S, d)$ such that $d \in S$ and $S \subseteq \mathbb{R}^{n}$ is compact, convex, and $d$-comprehensive. ${ }^{1,2}$ An element $(S, d) \in \overline{\mathcal{B}}$ is a bargaining problem if there is $s \in d$ with $s \gg d$. The set of all bargaining problems is denoted by $\mathcal{B} \subseteq \overline{\mathcal{B}}$.

The best that player $i$ can hope for in $(S, d)$ - her ideal payoff in $(S, d)$-is $a_{i}(S, d)=$ $\max \left\{s_{i}: s \in S\right.$ and $\left.s \geq d\right\}$. The point $a(S, d)=\left(a_{1}(S, d), \cdots, a_{n}(S, d)\right)$ (which is typically not feasible) is called the ideal point of $(S, d)$. The midpoint of $(S, d)$ is $m(S, d)=\frac{1}{n} a(S, d)$ and always lies in $S$ for $S$ is convex.

A bargaining solution with domain $\mathcal{B}^{\prime} \subseteq \overline{\mathcal{B}}$ is a map $F$ that assigns to each pair $(S, d) \in \mathcal{B}^{\prime}$ a point $F(S, d) \in S$. Normally, a bargaining solution is defined on a subset

\footnotetext{
${ }^{1}$ For $x, y \in \mathbb{R}^{n}$ we write $x \gg y$ if $x_{i}>y_{i}$ for all $i \in N$, we write $x \geq y$ if $x_{i} \geq y_{i}$ for all $i \in N$, and $x>y$ if $x \geq y$ and $x \neq y$.

${ }^{2} S$ is $d$-comprehensive if $x \in S$ for any $x$ with $d \leq x \leq s$ for some $s \in S$.
} 
of $\mathcal{B}$ rather than $\overline{\mathcal{B}}$. For reasons that will become clear later, it is beneficial to allow $F$ to operate on this larger set.

Throughout the paper we assume that bargaining solutions are translation invariant, that is for any $t \in \mathbb{R}^{n}$ it holds that $F(S+t, d+t)=F(S, d)+t$. In this case one can assume without loss of generality that $d=0$ as $F(S, d)=F(S-d, 0)+d$ for all bargaining problems $(S, d)$. In order to keep notation simple we will write $S$ for the problem $(S, 0)$.

Let $S$ be a problem. A point $s \in S$ is weakly Pareto optimal if there is no $t \in S$ with $t \gg s$, and strongly Pareto optimal if there is no $t \in S$ with $t>s$. Let $\operatorname{WPO}(S)$ and $\mathrm{PO}(S)$ denote the weak and, respectively, strong Pareto frontiers of $S$, that is the set of weak and, respectively, strong Pareto optimal points in $S$. Denote by $\mathcal{B}^{*}$ the collection of bargaining problems $S$ with $\mathrm{WPO}(S)=\mathrm{PO}(S)$.

\subsection{The Closure of $\mathcal{B}$}

Throughout the paper let $d_{H}$ denote the Hausdorff distance. ${ }^{3}$ The map $d_{H}$ is a pseudo-metric, but as $\mathcal{B}$ and $\overline{\mathcal{B}}$ contain only compact sets, the pairs $\left(\mathcal{B}, d_{H}\right)$ and $\left(\overline{\mathcal{B}}, d_{H}\right)$ are metric space. In particular, it is well known that $\mathcal{B}^{*}$ is a dense subset of $\mathcal{B}$ with respect to $d_{H}$. Let $\left(S^{p}\right)_{p \in \mathbb{N}} \subseteq \mathcal{B}$ be a Cauchy sequence of problems. ${ }^{4}$ If $S$ is an accumulation point of $\left(S^{p}\right)_{p \in \mathbb{N}}$ then $S$ is bounded, convex, comprehensive, and $0 \in S$. Moreover, for any two accumulation points $S, S^{\prime}$ one has $\bar{S}=\bar{S}^{\prime}$, that is $S$ and $S^{\prime}$ have identical closures. Hence, $\bar{S}$ is the unique accumulation point of $\left(S^{p}\right)_{p \in \mathbb{N}}$ in $\overline{\mathcal{B}}$. In particular, $\left(\overline{\mathcal{B}}, d_{H}\right)$ is a complete metric space and $\overline{\mathcal{B}}$ is the closure of $\mathcal{B}$.

In the following we shall formulate axioms for solutions on subsets of $\overline{\mathcal{B}}$, bearing in mind that not all elements of the respective domain are bargaining problems. We

\footnotetext{
${ }^{3}$ The Hausdorff distance between two subsets $X, Y$ of a metric space with metric $d$ is defined as $d_{H}(X, Y)=\max \left\{\sup _{x \in X} \inf _{y \in Y} d(x, y), \sup _{y \in Y} \inf _{x \in X} d(x, y)\right\}$.

${ }^{4}$ The sequence $\left(S^{p}\right)_{p \in \mathbb{N}}$ is a Cauchy sequence if for any $\varepsilon>0$ there is $p$ such that $d_{H}\left(S^{p^{\prime}}, S^{p^{\prime \prime}}\right)<\varepsilon$ for all $p^{\prime}, p^{\prime \prime} \geq p$.
} 
do so for technical reasons that will become clear later. However, the skeptical reader should rest assured that we do not impose our axioms anywhere outside $\mathcal{B}$.

\subsection{Standard Axioms}

For a permutation $\pi: N \rightarrow N$ let $\pi(S)=\left\{\left(s_{\pi(i)}\right)_{i \in N}: s \in S\right\}$. A problem is called symmetric if $\pi(S)=S$ for any permutation $\pi$. We start with three simple axioms a bargaining solution may satisfy.

Weak Pareto efficiency. A bargaining solution $F$ is weakly Pareto efficient on $\mathcal{B}^{\prime} \subseteq$ $\overline{\mathcal{B}}$ if $F(S)$ is weakly Pareto optimal in $S$ for each bargaining problem $S \in \mathcal{B}^{\prime}$.

Symmetry. A bargaining solution $F$ is symmetric on $\mathcal{B}^{\prime} \subseteq \overline{\mathcal{B}}$ if $F_{1}(S)=\ldots=F_{n}(S)$ for all symmetric bargaining problems $S \in \mathcal{B}^{\prime}$.

Continuity A bargaining solution $F$ is continuous on $\mathcal{B}^{\prime} \subseteq \overline{\mathcal{B}}$ if $\lim _{p \rightarrow \infty} F\left(S^{p}\right)=$ $F(S)$ for all sequences $\left(S^{p}\right)_{p \in \mathbb{N}} \subseteq \mathcal{B}^{\prime}$ and $S \in \mathcal{B}^{\prime}$ with $\lim _{p \rightarrow \infty} S^{p}=S$.

These three axioms are well known and have been discussed extensively in the bargaining literature (for instance in Peters, 1992), so we will keep this section short and emphasize only one point: continuity is typically only defined for sequences whose limit lies in $\mathcal{B}$, that is on $\mathcal{B}$ rather than on $\overline{\mathcal{B}}$. Requiring that it holds for all converging sequences - in particular those with limit in $\overline{\mathcal{B}} \backslash \mathcal{B}$-is a stronger requirement, which we will discuss in Subsection 5.1.

\section{Monotonicity and midpoint domination}

\subsection{Axioms}

Kalai (1977) introduced a very appealing notion of "fair" surplus division, by requiring that each player's share increase as the cake increases. More formally, he introduced the following axiom. 
Monotonicity. A bargaining solution is monotonic on $\mathcal{B}^{\prime} \subseteq \overline{\mathcal{B}}$ if $F(S) \leq F(T)$ for any two bargaining problems $S, T \in \mathcal{B}^{\prime}$ with $S \subseteq T$.

The most prominent monotonic solution is the egalitarian solution, E, that maps each problem $S$ to the weakly Pareto optimal point $E(S) \in S$ with $E_{i}(S)=E_{j}(S)$ for all $i, j \in N$. Hence, the arbitrator assigns the same (highest possible) payoff to all players.

Another very simple (although maybe not very convincing) way to arbitrate a problem $S$ is to randomly pick a player $i$ and allocate to her $a_{i}(S)$, whereas the remaining players obtain 0 . If each player is picked with equal probability, that is $\frac{1}{n}$, then player $i$ 's expected payoff is $m_{i}(S)$. Sobel (1981) introduced the view that a solution should be at least as favorable as this random assignment; formally, he introduced the following axiom.

Midpoint Domination. A bargaining solution $F$ is midpoint dominant on $\mathcal{B}^{\prime} \subseteq \overline{\mathcal{B}}$ if $F(S) \geq m(S)$ for all bargaining problems $S \in \mathcal{B}^{\prime}$.

There are well known bargaining solutions that satisfy this axiom, for instance those of Nash (1950) and Kalai and Smorodinsky (1975).5,6

\subsection{A Characterization and a Negative Result}

Midpoint domination alone is sufficient to characterize a bargaining solution on hyperplane problems, i.e. bargaining problems of the form $H=\operatorname{cch}\left\{\left\{a_{i} e^{i}\right\}_{i \in N}\right\}$ for some vector $a \in \mathbb{R}^{n} \cdot 7,8$ For these problems do not contain any point that weakly dominates their midpoint, one has $F(H)=m(H)$. We use this observation to show

\footnotetext{
${ }^{5}$ The Nash solution maps any problem $S$ to the unique maximizer of $\prod_{i \in N} x_{i}$ in $S$.

${ }^{6}$ The Kalai-Smorodinsky solution maps any problem $S$ to the intersection between $\operatorname{WPO}(S)$ and the line from 0 to $a(S)$.

${ }^{7} \mathrm{By} e^{i}$ we denote the $i$-th unit vector; that is $e_{i}^{i}=1$ and $e_{j}^{i}=0$ for all $i \in N$ and all $j \neq i$.

${ }^{8}$ For a set $X$ we denote by $\operatorname{cch}(X)$ the comprehensive convex hull of $X$, that is the smallest convex and comprehensive superset of $X$.
} 
that midpoint domination and monotonicity together uniquely define one bargaining solution.

Proposition 3.1. Let $\mathcal{B}^{\prime}$ be a set of bargaining problems that contains all hyperplane problems. A bargaining solution $F$ satisfies monotonicity and midpoint domination on $\mathcal{B}^{\prime}$ if and only if $F(S)=m(S)$ for all $S \in \mathcal{B}^{\prime}$.

Proof. Let $S$ be a problem, let $i \in N$, and for $\varepsilon>0$ define $S_{i}^{\varepsilon}$ as a hyperplane problem with $S \subseteq S_{i}^{\varepsilon}$ and $a_{i}\left(S_{i}^{\varepsilon}\right)=a_{i}(S)+\varepsilon$. (Since $S$ is compact and comprehensive such a problem always exists.) Then $F_{i}(S) \leq F_{i}\left(S_{i}^{\varepsilon}\right)=m_{i}\left(S_{i}^{\varepsilon}\right)=\frac{1}{n} a_{i}(S)+\frac{\varepsilon}{n}$ where the inequality comes from $F$ 's monotonicity. Since this is true for all $i \in N$ and all $\varepsilon>0$, we have $F(S) \leq m(S)$. By midpoint domination $F(S) \geq m(S)$, so $F(S)=m(S)$.

Unfortunately, the solution in Proposition 3.1 violates weak Pareto efficiency; so, this result should be interpreted as a negative one: there is no bargaining solution that satisfies weak Pareto efficiency, monotonicity, and midpoint domination.

\subsection{Reconciliation: Midpoint Monotonicity}

Our aim is to reconcile monotonicity and midpoint domination at least "in spirit": we keep midpoint domination, but we shall weaken monotonicity as much as necessary for the axioms not to conflict with weak Pareto efficiency. This leads to the following axiom.

Midpoint Monotonicity. A bargaining solution $F$ is midpoint monotonic on $\mathcal{B}^{\prime} \subseteq$ $\overline{\mathcal{B}}$ if $F(S) \leq F(T)$ for any two problems $S, T \in \mathcal{B}^{\prime}$ for which $S \subseteq T$ and there is $t \in T$ with $t \geq m(T)$ and $t \geq F(S)$.

In words, this axiom imposes monotonicity only if doing so causes no violation of midpoint domination. The following easy lemma is mainly stated for later reference. Nevertheless, it illustrates important cases in which this axiom has bite. 
Lemma 3.2. Let $F$ satisfy midpoint monotonicity, and let $S, T$ be bargaining problems with $S \subseteq T$. If $F(S) \geq m(T)$ then $F(T) \geq F(S)$.

Proof. Let $t=F(S)$. Since $t \in T, t \geq m(T)$, and $t \geq F(S)$, midpoint monotonicity applies, so $F(T) \geq F(S)$.

\section{The Midpoint-Constrained Egalitarian Solution}

Combining midpoint domination and midpoint monotonicity intuitively translates to finding a solution that satisfies midpoint domination and is, in some sense, as "close as possible" to a monotonic solution. Taking this aim literally, we define the solution $E^{*}$ as the solution of the minimization problem

$$
\begin{aligned}
& \min _{s \in S}\|s-E(S)\| \\
& \text { s.t. } s \geq m(S),
\end{aligned}
$$

where $\|$.$\| denotes the Euclidean norm. The constraints s \in S$ and $s \geq m(S)$ define a convex and compact feasibility set for any $S \in \overline{\mathcal{B}} \cdot{ }^{9}$ As the objective function in (1) is continuous, this optimization problem always has a solution. Moreover, the objective function is strictly quasi-convex. For if $s \neq t$ are such that $\|s-E(S)\|=\|t-E(S)\|$ then $\|\alpha s+(1-\alpha) t-E(S)\|<\|s-E(S)\|$ for all $\alpha \in(0,1)$. Hence, the solution of (1) is unique, which means that $E^{*}(S)$ is well-defined. We call this solution the midpoint-constrained egalitarian solution. Our main result is that $E^{*}$ satisfies our axioms - and no other bargaining solution does.

Theorem 4.1. A solution $F$ on $\mathcal{B}$ satisfies weak Pareto efficiency, symmetry, midpoint domination, and midpoint monotonicity if and only if $F=E^{*}$.

We postpone the proof of Theorem 4.1 to the end of Section 5.3. We shall first present an algorithm to compute $E^{*}$, from which we can derive an alternative, more useful,

\footnotetext{
${ }^{9}$ Note that we do not require that $s$ lie in the (weak or strong) Pareto frontier of $S$.
} 
representation of this bargaining solution. We will use the latter in order to prove that $E^{*}$ satisfies our axioms.

\subsection{Alternative representations of $E^{*}$}

Defining $E^{*}$ as the solution of a convex optimization problem is in line with our motivation to reconcile midpoint domination and (some form of) monotonicity. Nevertheless, in order to actually find $E^{*}(S)$ there are better ways. A simple procedure is the following: assign $E_{i}(S)$ to each player $i$. If $E(S) \geq m(S)$, then set $E^{*}(S)=E(S)$ and stop. Otherwise allocate to each $i \in N$ with $E_{i}(S)<m_{i}(S)$ the value $m_{i}(S)$, and allocate to all other players the (equal) payoff $r$ that is maximally feasible. Again, if the new allocation satisfies midpoint domination, stop; otherwise repeat the previous step. Clearly this procedure stops eventually (after no more than $n$ steps). Our first lemma formalizes this algorithm and shows that the resulting allocation is, in fact, $E^{*}(S)$.

Lemma 4.2. Let $S \in \mathcal{B}$. Let $I^{1}(S)=\left\{i \in M: E(S)<m_{i}(S)\right\}$. If $I^{1}(S)=\emptyset$, let $F(S)=E(S)$. Otherwise, define recursively

$$
\begin{aligned}
J^{t}(S) & =\bigcup_{k=1}^{t} I^{t}(S) \\
F^{t}(S) & =\max _{r:\left(\left(m_{j}(S)\right)_{j \in J^{t}(S)},(r)_{i \in N \backslash J^{t}(S)}\right) \in S}\left(\left(m_{j}(S)\right)_{j \in J^{t}(S)},(r)_{i \in N \backslash J^{t}(S)}\right) \\
I^{t+1}(S) & =\left\{i \in N \backslash J^{t}(S): F_{i}^{t}(S)<m_{i}(S)\right\} .
\end{aligned}
$$

Then, there is $t^{*} \leq n$ such that $F^{t}(S)=F^{t^{*}}(S)$ for all $t \geq t^{*}$. Moreover, $F^{t^{*}}(S)=$ $E^{*}(S)$.

Proof. The algorithm will stop after at most $n$ steps as in each non-terminal step $J^{t}$ increases by at least one player. So, $F^{t^{*}}(S)$ is well defined. Let $F=F^{t^{*}}(S)$ and assume that $F(S) \neq E^{*}(S)$ for some $S$; that is, $F$ does not solve (1). Let 
$\varepsilon=E^{*}(S)-F(S) \in \mathbb{R}^{n}$, and observe that for all $j \in J^{t^{*}}$

$$
F_{j}(S)+\varepsilon_{j}=E_{j}^{*}(S) \geq m_{j}(S)=F_{j}(S)
$$

Hence, $\varepsilon_{j} \geq 0$ for all $j \in J^{t^{*}}$ so that $\sum_{i \in N \backslash J^{t^{*}}} \varepsilon_{i} \leq 0$. Let $r=F_{i}(S)$ for $i \in N \backslash J^{t^{*}}$ (which is equal for all such $i$ ). By construction $r-E_{i}(S) \leq 0$, so the strict convexity of the square function implies $\sum_{i \in N \backslash J^{*}(S)}\left(r+\varepsilon_{i}-E_{i}(S)\right)^{2} \geq \sum_{i \in N \backslash J^{t^{*}}(S)}\left(r-E_{i}(S)\right)^{2}$. Hence,

$$
\begin{aligned}
\left\|E^{*}(S)-E(S)\right\|^{2} & =\|F(S)+\varepsilon-E(S)\|^{2} \\
& =\sum_{j \in J^{t^{*}}}\left(m_{j}(S)+\varepsilon_{j}-E_{j}(S)\right)^{2}+\sum_{i \in N \backslash J^{t^{*}}(S)}\left(r+\varepsilon_{i}-E_{i}(S)\right)^{2} \\
& \geq \sum_{j \in J^{t^{*}}}\left(m_{j}(S)-E_{j}(S)\right)^{2}+\sum_{i \in N \backslash J^{t^{*}}(S)}\left(r-E_{i}(S)\right)^{2} \\
& =\|F(S)-E(S)\|^{2} \\
& >\left\|E^{*}(S)-E(S)\right\|^{2},
\end{aligned}
$$

where the last inequality is true as $F$ does not solve (1). But this is impossible.

A second, very convenient, representation can now be obtained as an immediate corollary. (The proof is omitted.)

Corollary 4.3. Let $S \in \mathcal{B}$. Then $E^{*}(S)$ satisfies.

$$
E_{i}^{*}(S)= \begin{cases}m_{i}(S) & \text { if } m_{i}(S)>r \\ r & \text { otherwise }\end{cases}
$$

where $r=r(S)$ is maximal subject to $E^{*}(S) \in S$.

In the sequel we will refer to the number $r$ in Equation $(2)$ as $r(S)$. Hence, $E_{i}^{*}(S)=$ $\max \left\{m_{i}(S), r(S)\right\}$ for all $i \in N$. From here let $R(S)=\left\{i \in N: E_{i}^{*}(S)=r(S)\right\}$ and note that $R(S) \neq \emptyset$ for all $S \in \mathcal{B}$.

Kalai (1977) related his monotonic solution to the social welfare function of Rawls (1971), which maximizes the welfare of the least well off member of society. We can establish a similar connection between the latter and $E^{*}$. 
Corollary 4.4. Let $S \in \mathcal{B}$. Then

$$
E^{*}(S) \in \underset{s \in S: s \geq m(S)}{\arg \max } \min _{i \in N} s_{i}
$$

If $S \in \mathcal{B}^{*}$ then $\arg \max _{s \in S: s \geq m(S)} \min _{i \in N} s_{i}=\left\{E^{*}(S)\right\}$.

Proof. Let $S$ be a problem, let $x=E^{*}(S)$ and assume by contradiction that there exists a $y \in S$ that satisfies $y \geq m(S)$ and $\min _{i \in N} y_{i}>\min _{i \in N} x_{i}$. Then $y_{j} \geq$ $\min _{i \in N} y_{i}>\min _{i \in N} x_{i}=r(S)$ for all $j \in N$, contradicting the optimality of $r(S)$ in Equation (2). By construction any $s \in \arg \max _{s \in S: s \geq m(S)} \min _{i \in N} s_{i}$ must satisfy $s \geq E^{*}(S)$. Hence, if $S \in \mathcal{B}^{*}$ this set has a unique element, namely $E^{*}(S)$.

\subsection{Satisfied Axioms}

We start this section by proving that $E^{*}$ satisfies the properties in Theorem 4.1.

Lemma 4.5. $E^{*}$ satisfies weak Pareto efficiency, symmetry, midpoint domination, and midpoint monotonicity on $\mathcal{B}$.

Proof. For symmetric problems, one has $E^{*}(S)=E(S)$, which is symmetric. Weak Pareto efficiency is clear from Corollary 4.3, and midpoint domination follows from the constraint in (1). We show midpoint monotonicity. Let $S, T \in \mathcal{B}$ be such that $S \subseteq T$ and such that there is $t \in T$ with $t \geq m(T)$ and $t \geq E^{*}(S)$. Then $t_{i} \geq$ $\max \left\{m_{i}(T), r(S)\right\}$ for all $i \in N$. Let $t^{*}$ be the minimal such point. Note that if $r(S)>r(T)$ then $t_{i}^{*}=r(S)>r(T)$ for all $i \in R(T)$, contradicting the maximality of $r(T)$. So, $r(T) \geq r(S)$. Hence, $E_{i}^{*}(T)=\max \left\{m_{i}(T), r(T)\right\} \geq \max \left\{m_{i}(S), r(S)\right\}=$ $E_{i}^{*}(S)$.

We shall now turn to continuity, in particular continuity on $\overline{\mathcal{B}}$. For this purpose note that the egalitarian solution is well defined on problems $S \in \overline{\mathcal{B}} \backslash \mathcal{B}$, namely by $E(S)=0$. Thus, the optimization problem (1) has a unique solution on $S$ as well, namely $m(S)$, and $E^{*}$ is well defined on $\overline{\mathcal{B}}$ with $E^{*}(S)=m(S)$ for all $S \in \overline{\mathcal{B}} \backslash \mathcal{B}$. 
Lemma 4.6. The bargaining solution $E^{*}$ is continuous on $\overline{\mathcal{B}}$.

Proof. Let $\left(S^{p}\right)_{p \in \mathbb{N}} \subseteq \overline{\mathcal{B}}$ be a Cauchy sequence and let $S \in \overline{\mathcal{B}}$ with $\lim _{p \rightarrow \infty} S^{p}=S$. Note that any sequence $\left(s^{p}\right)_{p \in \mathbb{N}} \subseteq \mathbb{R}^{n}$ with $s^{p} \in S^{p}$ has a converging subsequence. Let $s^{p}=E^{*}\left(S^{p}\right)$, and let $\left(s^{p_{\ell}}\right)_{\ell \in \mathbb{N}}$ be a converging subsequence with $s^{p_{\ell}} \rightarrow s \in S$. Clearly $s \geq m(S)$ as $m($.$) is continuous and s^{p} \geq m\left(S^{p}\right)$ for all $p \in \mathbb{N}$ by construction. Assume that $s \neq E^{*}(S)$. Then there is $t \in S$ with $t \geq m(S)$ and $\|t-E(S)\|<$ $\|s-E(S)\|$. Define a sequence $\left(t^{p}\right)_{p \in \mathbb{N}}$ with $t^{p} \geq m\left(S^{p}\right)$ for all $p \in \mathbb{N}$, such that there is a converging subsequence $\left(t^{p_{\ell}}\right)_{\ell \in \mathbb{N}}$ with $t^{p_{\ell}} \rightarrow t$. Then

$$
\lim _{\ell \rightarrow \infty}\left\|t^{p_{\ell}}-E\left(S^{p_{\ell}}\right)\right\|=\|t-E(S)\|<\|s-E(S)\|=\lim _{\ell \rightarrow \infty}\left\|s^{p_{\ell}}-E\left(S^{p_{\ell}}\right)\right\| .
$$

In particular, for sufficiently large $\ell$, one has $\left\|t^{p_{\ell}}-E\left(S^{p_{\ell}}\right)\right\|<\left\|s^{p_{\ell}}-E\left(S^{p_{\ell}}\right)\right\|$, contradicting the definition of $s^{p_{\ell}}$ as the solution of the minimization problem (1).

\subsection{Characterization on $\mathcal{B}^{*}$}

In this subsection we shall prove that the characterization of Theorem 4.1 holds on $\mathcal{B}^{*}$. For the proof the following notation is needed: for $\theta, x \in \mathbb{R}_{\gg 0}^{n}$ let $\theta \circ x=\left(\theta_{i} x_{i}\right)_{i \in N}$. For a bargaining problem $S$ let $\theta \circ S=\{\theta \circ s: s \in S\}$, that is $\theta \circ S$ is a positive linear transformation of $S$ that keeps the origin fixed.

Proposition 4.7. A solution $F$ on $\mathcal{B}^{*}$ satisfies weak Pareto efficiency, symmetry, midpoint domination, and midpoint monotonicity if and only if $F=E^{*}$.

Proof. By Lemma 4.5 it is sufficient to show that $E^{*}$ is the only bargaining solution with these properties. So let $F$ be a solution that satisfies all four axioms. For a problem $S \in \mathcal{B}^{*}$ let $J(S)=J^{t^{*}}(S)$ be as in Lemma 4.2, and note that $J(S) \cup R(S)=N$ and $J(S) \cap R(S)=\emptyset$. We proceed via induction over $|J(S)|$, so suppose first that $J(S)=\emptyset$. Then $E^{*}(S)=E(S) \ll a(S)$ where the inequality is due to $S \in \mathcal{B}^{*}$. Let $\varepsilon=\min _{i \in N}\left(a_{i}(S)-E_{i}^{*}(S)\right)>0$ and let $T=\operatorname{cch}\left(\left\{\left(E_{i}^{*}(S)+\varepsilon\right) e^{i}\right\}_{i \in N} \cup\left\{E^{*}(S)\right\}\right)$. Then $T \subseteq S, T \in \mathcal{B}^{*}$, and $F(T)=E^{*}(S)$ by symmetry and weak Pareto efficiency. 
So, $F(T) \geq m(S)$ and $T \subseteq S$, so $F(S) \geq F(T)=E^{*}(S)$ by Lemma 3.2. Since $E^{*}(S)$ is strongly Pareto efficient (recall that $S \in \mathcal{B}^{*}$ ), we have $F(S)=E^{*}(S)$.

Let now $|J(S)| \geq 1$ and suppose the claim is true for all $S^{\prime}$ with $\left|J\left(S^{\prime}\right)\right| \leq|J(S)|-$ 1. Suppose without loss of generality that $J(S)=\{1, \ldots, k\}$, and that $k \in I^{t^{*}}(S)$ (that is, $k$ is added to $J(S)$ in the last non-trivial step of the algorithm). Let $\theta \in \mathbb{R}^{n}$ be defined by $\theta_{k}=\frac{n r(S)}{a_{k}(S)}$ and $\theta_{j}=1$ for all $j \neq k$. Let $S^{\prime}=\theta \circ S$ and note that $S^{\prime} \subseteq S$ as $\theta_{k}<1$, and that $S^{\prime} \in \mathcal{B}^{*}$. Let $x=\left(m_{1}(S), \cdots, m_{k-1}(S), r(S), \cdots, r(S)\right) \in S^{\prime}$. We show that $E^{*}\left(S^{\prime}\right)=x$ : let $z$ be the unique solution of (1) in $S^{\prime}$. Then $z_{i} \geq m_{i}\left(S^{\prime}\right) \geq$ $r(S)=x_{i}$ for all $i \in R(S) \cup\{k\}$, and $z_{j} \geq m_{j}\left(S^{\prime}\right)=x_{j}$ for all $j \in J(S) \backslash\{k\}$. Hence, $z \geq x$, and the strong Pareto optimality of $x$ requires $z=x$.

We now have $R\left(S^{\prime}\right)=R(S) \cup\{k\}$, that is $J\left(S^{\prime}\right)=J(S) \backslash\{k\}$. Hence, by the induction hypothesis, $F\left(S^{\prime}\right)=E^{*}\left(S^{\prime}\right) \geq m(S)$. By Lemma 3.2 midpoint monotonicity applies, so $F(S) \geq E^{*}\left(S^{\prime}\right)$. Since $F_{k}(S) \geq m_{k}(S)$ it follows that $F(S) \geq E^{*}(S)$, and strong Pareto optimality of the latter completes the proof.

\section{An Extension Theorem}

Let $F$ be a bargaining solution with domain $\mathcal{B}^{\prime}$, and let $\mathcal{B}^{\prime} \subseteq \mathcal{B}^{\prime \prime} \subseteq \overline{\mathcal{B}}$. A bargaining solution $\bar{F}: \mathcal{B}^{\prime \prime} \rightarrow \mathbb{R}^{n}$ is an extension of $F$ to $\mathcal{B}^{\prime \prime}$ if $\bar{F}(S)=F(S)$ for all $S \in \mathcal{B}^{\prime}$. It is easy to see that the egalitarian solution has a unique extension from $\mathcal{B}^{*}$ to $\mathcal{B}$ : For any $S \in \mathcal{B} \backslash \mathcal{B}^{*}$ can be approximated both from above and from below by arbitrarily close elements of $\mathcal{B}^{*}$, say $S^{\prime}$ and $S^{\prime \prime}$, so that $E\left(S^{\prime}\right) \leq F(S) \leq E\left(S^{\prime \prime}\right)$ for any monotonic $F$. In what follows we provide an extension theorem that formalizes and generalizes the above intuitive example; in particular, the theorem allows us to uniquely extend $E^{*}$ from $\mathcal{B}^{*}$ to $\mathcal{B}$ without imposing continuity. 


\subsection{Cauchy Regularity}

Let $(X, d)$ and $\left(Y, d^{\prime}\right)$ be two metric spaces. A map $f: X \rightarrow Y$ is Cauchy regular if for any Cauchy sequence $\left(x^{p}\right)_{p \in \mathbb{N}}$ the sequence $\left(f\left(x^{p}\right)\right)_{p \in \mathbb{N}}$ is a Cauchy sequence as well. Clearly any Cauchy regular map is continuous. The converse is not always true, but a sufficient condition is that $(X, d)$ is complete. This insight does not require a proof, and the following lemma is mainly stated for later reference.

Lemma 5.1. Let $(X, d)$ be a complete metric space, let $\left(Y, d^{\prime}\right)$ be a metric space, and let $f: X \rightarrow Y$ be continuous. Then $f$ is Cauchy regular.

Cauchy regular maps from a metric space $(X, d)$ into a complete metric space $\left(Y, d^{\prime}\right)$ have a unique continuous extension on the closure of $X$ (Spikes, 1981). We obtain a similar result for (weakly) monotonic extensions of (weakly) monotonic Cauchy regular maps in the next section, where we will precisely define what we mean by weakly monotonic. Bearing in mind that $E^{*}$ is a continuous map on the complete

metric space $\left(\overline{\mathcal{B}}, d_{H}\right)$, and therefore Cauchy regular, allows us then to uniquely extend $E^{*}$ from $\mathcal{B}^{*}$ to $\mathcal{B}$.

\subsection{Partially Ordered Metric Spaces}

A partially ordered metric space is a triple $(X, \succeq, d)$ such that $(X, d)$ is a metric space, and $\succeq$ is a partial order on $X$. Let $(X, \succeq, d)$ and $\left(Y, \succeq^{\prime}, d^{\prime}\right)$ be two partially ordered metric spaces. A map $f: X \rightarrow Y$ is called order-preserving if $f(x) \succeq^{\prime} f\left(x^{\prime}\right)$ for all $x, x^{\prime} \in X$ with $x \succeq x^{\prime}$.

Lemma 5.2. Let $(X, \succeq, d)$ and $\left(Y, \succeq^{\prime}, d^{\prime}\right)$ be two partially ordered metric spaces, such that $\left(Y, d^{\prime}\right)$ is complete. Let $D \subseteq X$ be a dense subset of $X$ such that for all $x \in X$ and all $\varepsilon>0$ there are $x^{\prime}, x^{\prime \prime} \in D$ with $d\left(x, x^{\prime}\right), d\left(x, x^{\prime \prime}\right) \leq \varepsilon$ and $x^{\prime} \preceq x \preceq x^{\prime \prime}$. Let $f: D \rightarrow Y$ be an order-preserving and Cauchy regular map. Then there is a unique order-preserving extension $\bar{f}$ of $f$ on $X$. 
Proof. Let $x \in X \backslash D$. Then, by the condition on $D$, there are sequences $\left(x^{p}\right)_{p \in \mathbb{N}}$ and $\left(z^{p}\right)_{p \in \mathbb{N}}$ in $D$ with $x^{p} \preceq x \preceq z^{p}$ for all $p$, and $\lim _{p \rightarrow \infty} x^{p}=\lim _{n \rightarrow \infty} z^{p}=x$. As $f$ is Cauchy regular both $\left(f\left(x^{p}\right)\right)_{p \in \mathbb{N}}$ and $\left(f\left(z^{p}\right)\right)_{p \in \mathbb{N}}$ are Cauchy sequences, and as $(Y, d)$ is complete, they are converging. Suppose that there is an order-preserving extension $\bar{f}$ of $f$. Then $\lim _{p \rightarrow \infty} f\left(x^{p}\right) \preceq \bar{f}(x) \preceq \lim _{p \rightarrow \infty} f\left(z^{p}\right)$. Since the sequence $\left(w^{p}\right)_{p \in \mathbb{N}}$ with $w^{2 p-1}=x^{p}$ and $w^{2 p}=z^{p}$ is a Cauchy sequence as well, so is $\left(f\left(w^{p}\right)\right)_{p \in \mathbb{N}}$. Hence, $\lim _{p \rightarrow \infty} f\left(x^{p}\right)=\lim _{p \rightarrow \infty} f\left(w^{p}\right)=\lim _{p \rightarrow \infty} f\left(z^{p}\right)$, and $\bar{f}(x)=\lim _{p \rightarrow \infty} f\left(x^{p}\right)$. It remains to show that there is an order-preserving extension $\bar{f}$. For this purpose it is sufficient to show that $\bar{f}(x)$ as constructed above is well-defined, i.e. does not depend on the choice of $\left(x^{p}\right)_{p \in \mathbb{N}}$. So, let $\left(\hat{x}^{p}\right)_{p \in \mathbb{N}}$ be another sequence with $\hat{x}^{p} \rightarrow x$.

Clearly $\left(f\left(\hat{x}^{p}\right)\right)_{p \in \mathbb{N}}$ converges. Let $\left(\hat{w}^{p}\right)_{p \in \mathbb{N}}$ be defined analogously to $\left(w^{p}\right)_{p \in \mathbb{N}}$. Then $\lim _{p \rightarrow \infty} f\left(\hat{x}^{p}\right)=\lim _{p \rightarrow \infty} f\left(\hat{w}^{p}\right)=\lim _{p \rightarrow \infty} f\left(z^{p}\right)=\bar{f}(x)$.

\subsection{Weakly Monotonic Bargaining Solutions}

In order to define any kind of monotonicity for a bargaining solution, (partial) orders on $\mathbb{R}^{n}$ and on the set of bargaining problems are required. We always equip $\mathbb{R}^{n}$ with the partial order $\geq$ and the Euclidian metric.

Weak Monotonicity A bargaining solution $F$ is weakly monotonic if there is a partial order $\succeq$ on $\mathcal{B}$ such that $F$ is order-preserving.

As an illustration observe that any monotonic bargaining solution is weakly monotonic: define $S \preceq T$ if and only if $S \subseteq T$. Similarly, a bargaining solution is restricted monotonic (see for instance Roth, 1979) if and only if it is weakly monotonic with respect to the order $\preceq$ such that $S \preceq T$ whenever $S \subseteq T$ and $a(S)=a(T)$. As each individually monotonic bargaining solution (Kalai and Smorodinsky, 1975) is also restricted monotonic (Peters and Tijs, 1985), they are weakly monotonic as well.

Recall from Section 2.2 that $\left(\mathcal{B}, d_{H}\right)$ is a metric space and that $\left(\overline{\mathcal{B}}, d_{H}\right)$ is a complete metric space. The following theorem is now clear. 
Theorem 5.3. Let $F$ be a bargaining solution that is continuous and weakly monotonic on $\overline{\mathcal{B}}$, and let $G$ be the restriction of $F$ on $\mathcal{B}^{*}$. Let $\bar{G}$ be a weakly monotonic extension of $G$ on $\mathcal{B}$. If for each bargaining problem $S \in \mathcal{B}$ and each $\varepsilon>0$ there are problems $S^{\prime}, S^{\prime \prime} \in \mathcal{B}^{*}$ with $d_{H}\left(S, S^{\prime}\right), d_{H}\left(S, S^{\prime \prime}\right) \leq \varepsilon$ and $S^{\prime} \preceq S \preceq S^{\prime \prime}$ then $\bar{G}(S)=F(S)$ for all $S \in \mathcal{B}$.

Proof. As $\left(\overline{\mathcal{B}}, d_{H}\right)$ is a complete metric space, $F$ is Cauchy regular by Lemma 5.1. Hence, $\bar{G}$ is an order-preserving extension of an order-preserving and Cauchy-regular map from $\mathcal{B}^{*}$ to $\mathcal{B}$. As $\mathcal{B}^{*}$ is a dense subset of $\mathcal{B}$ and each element can be approximated from above and from below by a sequence in $\mathcal{B}^{*}$, Lemma 5.2 applies and there is a unique weakly monotonic extension. As $F$ is a weakly monotonic extension, $\bar{G}$ and $F$ must coincide on $\mathcal{B}$.

In order to complete the proof of Theorem 4.1 it is now sufficient to show that $E^{*}$ is weakly monotonic and that each problem in $\mathcal{B}$ can be approximated from below and from above by sequences in $\mathcal{B}^{*}$. For this purpose define the partial order $\preceq$ as

$$
S \preceq T \text { if and only if }\left\{\begin{array}{l}
S \subseteq T, \text { and there is } t \in T \text { with } \\
t_{i} \geq \max \left\{E_{i}^{*}(S), m_{i}(T)\right\} \text { for all } i \in N .
\end{array}\right.
$$

Proof of Theorem 4.1. $E^{*}$ is order-preserving with respect to $\preceq$, and Cauchy regular by Lemmas 4.6 and 5.1 .

We first show that an approximation from below (in the sense of Theorem 5.3) exists, so let $S \in \mathcal{B} \backslash \mathcal{B}^{*}$. Let $S^{0}=\operatorname{cch}\left(\left\{a_{i}(S) e^{i}\right\}_{i \in N}\right)$. Define recursively for each $s \in \mathrm{WPO}(S)$ and each integer $p \geq 0$ the point $t^{p}(s)$ as the intersection of the line from 0 to $s$ with $\mathrm{WPO}\left(S^{p}\right)$. Let $f^{p+1}: \mathrm{WPO}(S) \rightarrow S$ be defined as $f^{p+1}(s)=\frac{1}{2}\left(s+t^{p}(s)\right)$, and let $S^{p+1}=\operatorname{cch}\left(f^{p+1}(\mathrm{WPO}(S))\right)$. Clearly, $S^{p} \subseteq S^{p+1}$ for all $p \geq 0$, and $S^{p} \rightarrow S$. Hence, for each $\varepsilon>0$ there is $p$ such that $d_{H}\left(S^{p}, S\right) \leq \varepsilon$ for all $p^{\prime} \geq p$. Since $S^{p} \subseteq S$ and $E^{*}\left(S^{p}\right) \geq m\left(S^{p}\right)=m(S)$, one obtains $S^{p} \preceq S$ for all $p$ by Lemma 3.2. It remains to show that $S^{p} \in \mathcal{B}^{*}$ for all $p \geq 0$. This is true for $S^{0}$ by construction, so suppose it is true for some $p \geq 0$. Let $\bar{s}^{1}, \bar{s}^{2} \in \mathrm{WPO}(S)$, let $s^{1}=f^{p+1}\left(\bar{s}^{1}\right)$ and $s^{2}=f^{p+1}\left(\bar{s}^{2}\right)$, and 
assume that $s^{1}>s^{2}$. Let $\lambda^{1}, \lambda^{2} \in(0,1)$ be such that $t^{p}\left(\bar{s}^{1}\right)=\lambda^{1} s^{1}$ and $t^{p}\left(\bar{s}^{2}\right)=\lambda^{2} s^{2}$. As $t^{p}\left(\bar{s}^{2}\right)$ is strongly Pareto efficient in $S^{p}$ (recall that $S^{p} \in \mathcal{B}^{*}$ by the induction hypothesis) and $\lambda^{2} s^{1}>\lambda^{2} s^{2}=t^{p}\left(\bar{s}^{2}\right)$, it must hold that $\lambda^{1}<\lambda^{2}$. In particular $s^{1}-t^{p}\left(\bar{s}^{1}\right)=\left(1-\lambda^{1}\right) s^{1} \gg\left(1-\lambda^{2}\right) s^{2}=s^{2}-t^{p}\left(\bar{s}^{2}\right)$. From the construction of $s^{1}$ and $s^{2}$ we have $\bar{s}^{1}=s^{1}+\left(s^{1}-t^{p}\left(\bar{s}^{1}\right)\right)$ and $\bar{s}^{2}=s^{2}+\left(s^{2}-t^{p}\left(\bar{s}^{2}\right)\right)$. Therefore, $\bar{s}^{1} \gg \bar{s}^{2}$, contradicting $s^{2} \in \mathrm{WPO}(S)$. So, $S^{p} \in \mathcal{B}^{*}$ for all $p \geq 0$.

We next prove that $S$ can be approximated from above. First note that $m(S) \notin$ $\mathrm{WPO}(S)$ as $S \notin \mathcal{B}^{*}$. Let $M(S)=\left\{i \in N: E_{i}^{*}(S)>m_{i}(S)\right\} \subseteq R(S)$. Then $M(S) \neq \emptyset$ as $E^{*}$ is weakly Pareto efficient. Let $\varepsilon>0$ and assume without loss of generality that $\varepsilon \leq n \cdot \min _{i \in M(S)}\left(E_{i}^{*}(S)-m_{i}(S)\right)$. Define $S^{\varepsilon}=\operatorname{cch}\left(S \cup\left\{\left(a_{i}(S)+\varepsilon\right) e^{i}\right\}_{i \in M(S)}\right)$. Then, by construction, $m\left(S^{\varepsilon}\right) \leq E^{*}(S)$. Hence $S \preceq S^{\varepsilon}$. Moreover, $d_{H}\left(S, S^{\varepsilon}\right) \leq \varepsilon$.

So, all premises of Theorem 5.3 are satisfied, that is $E^{*}$ is the unique bargaining solution that satisfies weak Pareto efficiency, symmetry, midpoint domination, and midpoint monotonicity on $\mathcal{B}$.

\section{Discussion}

The strength of Theorem 5.3 lies in the fact that a continuity axiom is not needed: if a continuous and weakly monotonic extension is known then it must be unique. A word of caution is appropriate though: The extension $\bar{G}$ is unique only on $\mathcal{B}$, but not on $\overline{\mathcal{B}}$, for elements of $\overline{\mathcal{B}} \backslash \mathcal{B}$ cannot be approximated from below by elements of $\mathcal{B}^{*}$. As a simple example let $\bar{G}$ be such that $\bar{G}(S)=0$ for all $S \in \overline{\mathcal{B}} \backslash \mathcal{B}$. This is another monotonic extension of $E^{*}$ from $\mathcal{B}^{*}$ to $\overline{\mathcal{B}}$.

The axioms we have used in the characterization are independent: The solution $F(S)=m(S)$ satisfies all axioms but Pareto efficiency, the egalitarian solution satisfies all axioms but midpoint domination, and the Nash solution satisfies all axioms but midpoint monotonicity. Efficiency, midpoint domination, and midpoint monotonicity are satisfied by the solution $F^{q}$ with $F_{i}^{q}(S)=\max \left\{m_{i}(S), t(S) q_{i}\right\}$ for all 
$i \in N$, where $q \in \mathbb{R}_{\gg 0}^{n}$ and $t(S)$ such that $F^{q}(S) \in \operatorname{WPO}(S)$. Symmetry is then violated whenever $q$ is not symmetric.

\section{References}

Kalai, E., 1977. Proportional solutions to bargaining situations: Interpersonal utility comparisons. Econometrica 45, 1623-1630.

Kalai, E., Smorodinsky, M., 1975. Other solutions to Nash's bargaining problem. Econometrica 43, 513-518.

Luce, R.D., Raiffa, H., 1957. Games and Decisions: Introduction and Critical Survey. John Wiley and Sons.

Moulin, H., 1983. Le choix social utilitariste. Ecole Polytechnique Discussion Paper.

Nash, J., 1950. The bargaining problem. Econometrica 18, 155-162.

Peters, H., 1992. Axiomatic Bargaining Theory. Kluwer Academic Publishers.

Peters, H.J., Tijs, S., 1985. Characterization of all individually monotonic bargaining solutions. The International Journal of Game Theory 14, 219-228.

Rachmilevitch, S., 2014. Randomized dictatorship and the Kalai-Smorodinsky bargaining solution. Theory and Decision 76, 173-177.

Rawls, J., 1971. A Theory of Justice. Belknap.

Roth, A.E., 1979. Axiomatic Models of Bargaining. Springer.

Sobel, J., 1981. Distortion of utilities and the bargaining problem. Econometrica 49, $597-619$.

Spikes, R.F., 1981. Cauchy-regular functions. Journal of Mathematical Analysis and Applications 79, 18-25. 